\title{
A produção científica brasileira sobre a Estratégia Saúde da Família ea mudança no modelo de atenção
}

\author{
Brazilian scientific production on the Family Health Strategy \\ and the change in the model of care
}

Livia Angeli Silva ${ }^{1}$

Cezar Augusto Casotti ${ }^{1}$

Sônia Cristina Lima Chaves ${ }^{2}$

${ }^{1}$ Universidade Estadual do Sudoesteda Bahia. Av. David Fadini SN, Rosa N eto. 45820-000 Eunapolis BA. schaves@ufba.br

${ }^{2}$ Departamento de Odontologia Social, Faculdade deOdontologia, Universidade Federal da Bahia.
Abstract The variety of existing studies addressing the Family Health Strategy (ESF) and its role in the reorientation of the model of care in the country has motivated the systematization of the advances and limitations described in these studies. Thus, thisstudy, from a review of thescientific production on this theme, analyzed if the ESF has been able to modify the model of care. Forty-one articlesfrom theScielo database published between 2002 and 2010 that fulfilled the inclusion criteria were analyzed. They were fully analyzed in terms of political-institutional, organizational or technical-care dimensions. The results show that despite the improvement in the work process in primary care, its replacement aspect was not seen in themajority of the studies. Universal expansion of access to the health services, extension of coverage and focus were predominant. The changes are observed when analyzed in light of the demand, with better attendance and connectivity. The most evident limitsarein thelack of focus on health needs, as in the territorial issue, community participation and the social determinants addressed in an intersectoral way. Different degrees of the implementation of strategy were identified, though they have not yet resulted in system reorganization at local level.

Key words Family Health Program, Health care, $\mathrm{H}$ ealth evaluation
Resumo A variedade de estudos existentes abordando a Estratégia Saúde da Família e seu papel na reorientação do modelo de atenção no país motivou a sistematização dos avanços e limites descritos nesses estudos. Assim, este estudo, a partir de uma revisão da produção científica sobre o tema, analisou se a ESF tem sido capaz de modificar o modelo de atenção. Foram encontrados quarenta eum artigos da base de dados Sciel o publicados entre 2002 e 2010 que preencheram os crité rios deinclusão. Estes foram analisados na íntegra nas dimensões político-institucional, organizacional ou técnico-assistencial. O s resultados mostram queapesar da melhoria do processo detrabalho na atenção primária, seu caráter substitutivo não foi evidenciado na maioria dos estudos. Foi predominantea expansão da universalização do acesso aos serviços de saúde, a extensão de cobertura e focalização. Asmudanças são verificadas quando analisadas sob o foco da demanda, como maior acolhimento e vínculo. Os limites mais evidentes se situam no pouco foco nas necessi dades de saúde, como na territorialização, participação comunitária e enfrentamento dos determinantes sociais de forma intersetorial. Foram verificados diferentes graus de implantação da estratégia, mas que ainda não resultou na reorganização do sistema no nível local.

Palavras-chave Programa Saúde da Família, Atenção à saúde, Avaliação em saúde 
Introdução

A implementação do Sistema Único de Saúde (SUS) no Brasil, impulsionado pelo M ovimento da Reforma Sanitária, trouxe como um dos pontos centrais de suas proposições a necessidade da mudança no modelo de atenção. Inicialmente, na primeira década da implantação, a Atenção Primária à Saúde, designada nacionalmente de Atenção Básica, foi considerada como caminho para alcançar a universalidade das ações no território nacional ${ }^{1}$. No entanto, naquela conjuntura, prevaleceu a concepção de atenção primária seletiva, com um conjunto de ações limitadas², muitas vezes focadas no controle de doenças mais prevalentes e, durante algum tempo, os debates sobre as práticas de saúde, na pauta das políticas, deram lugar àqueles voltados à gestão e ao financiamento ${ }^{3}$.

Considerando M odelo deA tenção na perspectiva de Paim" 4 como "conteúdo" dos sistemas de saúde, o foco da mudança seria exatamenteas práticas, uma vez que, em seu conceito, o modelo éa forma de se combinar as tecnologias para a resolução deproblemas eatendimento àsnecessidades de saúde tanto individuais quanto coletivas.

Nesse sentido, para o desenvolvimento dessas práticas, as dimensões política, organizacional e técnico-assistencial devem ser consideradas ${ }^{3}$. A política diz respeito aos mecanismos de condução do processo de reorganização dos serviços, a organizacional se refereà relação entre as unidades de prestação de serviços e a técnicoassistencial perpassa pelas relações estabelecidas entre sujeitos e objetos de trabalho.

No sentido da reestruturação dos serviços de saúde a partir do SUS, foi criado, em 1994, o Programa Saúde da Família (PSF), numa perspectiva focalizadora, a partir do qual, buscavase superar desigualdades no acesso aos serviços de saúde e visava-se 0 al cance da equidade dentro do sistema².

Por apresentar características distintas dos demais programas e por ter sido considerado um espaço de reorganização do processo de trabalho em saúde no nível da atenção básica, o PSF foi considerado, em 1997, uma estratégia possível para reorientação daAtenção Básica e, consequentemente, do modelo de atenção à saúde no país $5^{5}$.

Neste sentido, o processo de trabalho da Estratégia Saúde da Família (ESF) deve se caracterizar, segundo o documento oficial que a instituiu e mais recentemente pela atual Política da Atenção Básica, pelo desenvolvimento de ações multidisciplinares, planejadas a partir das neces- sidades locais, por meio do diagnóstico de saúde do território e estabelecimento de vínculos entre profissionais e população, com fins de garantir a efetividade da Atenção Primária ${ }^{5,6}$.

Entretanto, para que a ESF se constitua em proposta alternativa de modelo de atenção, ela precisa ter uma combinação de tecnologias euma correlação deforças favoráveis às mudanças, caso contrário, continuará sendo o PSF em sua conformação inicial, como mais um programa verticalizado4. Além disso, suas ações deveriam se caracterizar pela intersetorialidade, educação permanente, monitoramento, avaliação e estímulo à partici pação popular, visando a uma melhoria gradativa nas condições de vida da população ${ }^{5}$.

Em diferentes momentos, desde a implantação da ESF, alguns estudos têm apontado para o distanciamento entre as práticas recomendadas daquelas que vêm sendo implementadas em diversos cenários ${ }^{2,3,7}$. Alguns autores têm sinalizado sua incompletude no quediz respeito à alteração do modelo hegemônico, uma vez que tem sido insuficientea mudança na forma de pensar e fazer no cotidiano das ações nos diversos níveis ${ }^{7.8}$.

As análises de implantação evidenciam que pela própria característica do programa inicialmente focalizado e somado às intensas diferenças regionais do país, a implementação da ESF, a partir de 1998, aconteceu em contextos diversificados e com intensidades e características também muito diferenciadas. Inicialmente sua implantação acorreu de forma mais intensa em municípios de grande porte, mas sem atingir boa cobertura. Posteriormente, inicia-se a implantação em municípios menores e, neste caso, esta implantação veio acompanhada de expansão da proporção de cobertura potencial9.

Esta, também foi diferenciada, tanto em relação ao ritmo quanto à proporção, entre as regiões brasileiras e entre os grupos de municípios com maior ou menor renda per capita ${ }^{10}$. Além disso, a avaliação normativa, realizada pelo $\mathrm{M} \mathrm{i-}$ nistério da Saúde, referente ao período de 1998 a 2002, revela diferenças também na estrutura eno processo de trabal ho entre os municípios e entre as regiões do país ${ }^{11}$. Tais diferenças dizem respeito à disponibilização de equipamentos, formas de contratação, remuneração, utilização de mapas da área, realização de reuniões com a comunidade, atendimento de enfermagem, ações de vigilância epidemiológica, capacitação da equipe, apoio diagnóstico e referência.

Nesse contexto, foi implementado o Projeto de Expansão e Consolidação Saúde da Família (PROESF), frente à realidade da baixa expansão 
decobertura potencial da ESF nos municípios de grande porte ${ }^{2}$. Esse projeto proporcionou um incremento no financiamento daESF nos municípios com população acima de 100 mil habitantes. Por se tratar de um projeto amplo que envolveu órgãos internacionais, ele impulsionou os estudos avaliativos, inclusive meta-avaliação seguindo padrões internacionais ${ }^{12}$.

Entreosanos de 2000 e2006, o M inistério da Saúdefinanciou estudos de avaliação daESF, nos quais muitos compuseram os Estudos de Linhas de Base do PROESF. H ouvetambém um aumento no número de teses, dissertações e outros projetos desenvolvidos por Universidades ${ }^{13}$.

Contudo, muitos destes estudos não empregaram a mesma metodologia, o que apontou formas distintas de apresentação dos resultados $^{14}$. Destaca-se ainda a concentração de pesquisas em alguns estados e a ausência total em outros, bem como o grande número de municípios caracterizados como insatisfatórios em re lação aos processos de trabalho, contrastando com resultados satisfatórios no que tange à meIhoria de indicadores e satisfação de usuários.

Assim, nesses doze anos de implantação da ESF, ocorreram mudanças si gnificativas nas práticas em saúde no âmbito da Atenção Básica, em muitas localidades, evidenciadas pelo incremento de ações de vigilância à saúde, estabelecimento de vínculos, o que pode ser observado pela avaliação que os próprios profissionais e usuários fazem do trabalho.

No entanto, nos municípios de grande porte, houve uma estagnação na expansão da ESF, sendo cada vez mais evidentes os problemas relacionados à reestruturação da rede de serviços como: acesso aos serviços especializados, referência e contrareferência, apoio diagnóstico eo não enfrentamento dos Determinantes Sociais em Saúde.

De acordo com o exposto e considerando as dimensões política, técnico-assistencial eorganizacional que envolvem as práticas de saúde das quais dependem a mudança no modelo de atenção, percebe-se a presença de elementos importantes na maioria dos estudos que avaliam/analisam a ESF. Todavia, a própria concepção de modelo não é uniformenem o método utilizado nas avaliações. Além disso, os componentes do processo de trabalho aparecem de forma diversificada nos diferentes desenhos de estudo. N esse caso, não se evidenciam quais desses componentes têm se modificado no sentido de alterar o modelo.

Considerando essa realidade multifacetada, bem como a variedade de modalidades de estu- dos realizados, este artigo visou identificar, a partir de uma revisão da produção científica, se a ESF tem sido capaz de modificar o modelo de atenção, no sentido das práticas de saúde, equais são as mudanças observadas no seu "núcleo duro" ou conteúdo do sistema.

\section{Metodologia}

Foi realizada revisão da produção científica de periódicos indexados à base de dados Scielo, referente à Saúde da Família no Brasil, desde sua implantação em 1998 até 2010. Em virtude da restrição nas buscas quando utilizados descritores mais específicos como "modelo de atenção" ou "modelo assistencial", optou-se pelos descritores "Programa Saúde da Família” e "Estratégia Saúde da Família". Retornaram da busca 771 e 420 publicações, respectivamente, e, partindo desses descritores, a seleção foi feita por meio da leitura dos resumos dos trabalhos.

Foram incluídos os artigos nos quais os resumos falavam da mudança do modelo ou que se referiam ao processo de trabalho ou às práticas de saúde, no sentido como fomulado por Paim4. Foram excluídos os de reflexão teórica, notas técnicas, resultados de pesquisas de concepções ou percepções de profissionais egestores e aqueles que tratavam de programas ou práticas de categorias profissionais específicas. Logo, para fins deste estudo, foram considerados apenas os estudos empíricos que anal isassem o processo detrabal ho/práticas em saúde da ESF como um todo. Do total, 41 trabalhos foram selecionados, lidos e analisados na íntegra.

Para fundamentação da análise, o conceito de modelo de atenção utilizado no presente estudo foi aquele desenvolvido por Paim ${ }^{4}$, efoi considerada a divisão proposta quanto ao foco que os modelos podem adotar, quais sejam, podem estar voltados para a demanda, centrados no atendimento ao que surge espontaneamente ou o que são induzidos pela oferta. M as numa outra perspectiva, podem estar centrados na identificação de necessidades, sendo estas de saúde ou sociais que não necessariamente se expressam em demanda.

$\mathrm{Na}$ análise do material encontrado, inicialmente foi feita uma caracterização dos estudos. Nesse sentido foram trabalhadas as variáveis/ categorias: ano de publicação, ano de desenvolvimento da pesquisa, relação com o PROESF, cenários de estudo, porte dos municípios estudados, fontes de dados e sujeitos da pesquisa. 
Um mesmo artigo poderia ser classificado em mais de uma categoria de análise.

No segundo momento, buscou-se identificar de que maneira a mudança do modelo de atenção foi descrita nos diversos estudos. Assim, foram analisadas as questões de pesquisa de cada estudo e o referencial teórico utilizado, observando-se se trazia um conceito de Modelo de Atenção, qual concepção predominante e qual a concepção do Programa Saúde da Família.

A partir das questões de pesquisa, foi realizado um agrupamento dos estudos que permitiu uma maior aproximação com os objetos trabaIhados em cada um deles e seus respectivos re sultados. Os estudos analisados foram categorizadosem 4 grupos: 1) Análise de implantação da ESF; 2) Avaliação/análise do processo de trabaIho; 3) Estudos comparativose4) Análise da rede de atenção.

$\mathrm{N}$ a última etapa foram identificados os componentes das práticas avaliadas e feito um agrupamento de acordo com as dimensões políticoinstitucional, organizacional ou técnico-assistencial, conforme concepção sistêmica de Teixeira ${ }^{3}$. N essemomento, foi identificado em quemedida esses componentes se constituíam em mudanças positivas ou como limites para a mudança etambém a quais cenários se referiam.

Para fins desta sistematização e cálculo das frequências simples, esses dados foram analisados utilizando o programa Epi info versão 3.3. Análises estatísticas não foram realizadas.

\section{Resultados}

Os quarenta e um estudos revisados ${ }^{15-55}$ apontam mudanças positivas no processo de trabaIho na ESF, mas também revelam limitações que comprometem a implementação da estratégia tal como foi preconizada. As mudanças têm ocorrido em práticas isoladas ${ }^{15,16}$ ou até mesmo em conjunto com uma das três dimensões ${ }^{17,18}$, mas não são capazes de alterar o modelo de atenção. A maior parte dos estudos apresenta mudanças parciais no modelo vigente, alguns ainda apresentam a implantação de um "programa parale$10^{\prime 19}$, sendo poucos os municípios nos quais a ESF realmente tenha desencadeado a reorganização do sistema de saúde (Tabelas 1, 2 e 3).

0 caráter substitutivo da ESF não foi evidenciado na mai oria dos estudos. Foi predominante o caráter da universalização do acesso aos serviços de saúde ${ }^{20}$, a extensão de cobertura ${ }^{21}$ e a focalização ${ }^{19}$.
Tomando como referência o modelo focado nas necessidades, os indícios de mudanças são ainda menores. Os avanços descritos apontam mais no sentido da prática assistencial relacionada ao acolhimento ${ }^{16}$, ao vínculo ${ }^{17,18}$, às orientações fornecidas ao usuário ${ }^{22}$, ou seja, ao componente demanda. Ainda são pouco exploradas as ações essenciais que elevam a ESF à condição de reorientadora do modelo como a exploração do território, a educação em saúde, a participação popular, o planejamento e a avaliação, ou seja, aquelas focadas nas necessidades.

$\mathrm{Na}$ dimensão organizacional, vale destacar a desarticulação da rede de atenção ${ }^{19}$ e na dimensão político-institucional, a ausência de políticas públicas que enfrentem os determinantes sociais numa perspectiva intersetorial ${ }^{23}$. Esses fatores interferem diretamente na garantia da integralidade tanto no que diz respeito ao acesso a todos os níveis de atenção como também à integração de práticas de promoção, prevenção e recuperação.

\section{Caracterização dos estudos encontrados}

A publicação de estudos dessa natureza iniciou-se na base de dados analisada a partir de 2002, mas essa produção só aumenta a partir de 2006. Observa-se que muitos desses estudos es-

Tabela 1. Distribuição numérica dos artigos sobre a Estratégia Saúde da Família e seu papel na reorientação do modelo de atenção identificados na base de dados scielo, no período de 2002 a 2010, segundo o tipo de estudo e/ou objeto estudado.

Grupo de análise por tipo de estudo/ objeto estudado

1) Análise de implantação da ESF

Estudos de Linhas de Base

Estudos qualitativos descritivos

Avaliação de grau de implantação

2) Avaliação/análise do processo detrabalho

A colhimento

Vínculo

Trabalho em equipe

Assistência domiciliar

Integralidade

Participação popular

Educação em saúde

Gerência

3) Estudos comparativos

USF X UBS tradicional

4) Análise da rede de atenção

Acesso a rede

Referência e contra-referência

\section{$\mathrm{n}$} 06

02

03

02

02

01 
Tabela 2. Distribuição numérica dos artigos sobre a Estratégia Saúde da Família e seu papel na reorientação do modelo de atenção identificados no Scielo entre 2002 e 2010, em categorias que descreveram mudanças positivas do modelo de atenção, segundo as dimensões político-institucional, organizacional e técnico-assitencial, local do estudo e porte do município.

\begin{tabular}{|c|c|c|c|c|c|c|}
\hline \multirow[b]{2}{*}{ Dimensão/Categoria } & \multicolumn{3}{|c|}{ Local do estudo } & \multicolumn{3}{|c|}{ Porte do município* } \\
\hline & USF & $\begin{array}{c}1 \text { ou } 2 \\
\text { municípios }\end{array}$ & $\begin{array}{c}3 \text { ou mais } \\
\text { municípios }\end{array}$ & Pequeno & Médio & Grande \\
\hline \multicolumn{7}{|l|}{ Político-institucional } \\
\hline Tipo de Gestão (participativa) & - & 01 & 01 & 01 & 02 & 01 \\
\hline Política de pessoal (estratégias de fixação) & - & - & 03 & - & - & 03 \\
\hline Financiamento (disponibilidade de recursos) & - & - & 01 & - & 01 & 01 \\
\hline Capacidade de governo (Qualificação dos gestores) & - & - & 02 & - & & 02 \\
\hline Capacidade instalada & - & 01 & 04 & 01 & 02 & 05 \\
\hline Ações intersetoriais como política de governo & - & - & 02 & - & - & 02 \\
\hline Projeto de governo ( Priorização da ESF ) & - & 02 & 03 & 01 & 02 & 03 \\
\hline \multicolumn{7}{|l|}{ Organizacional } \\
\hline Acesso à Atenção Básica & 01 & 02 & 04 & 01 & 01 & 07 \\
\hline Integralidade (acesso aos níveis secundário e terciário) & - & - & 01 & - & & 01 \\
\hline Articulação da rede de atenção & - & - & 04 & - & - & 04 \\
\hline Controle social no âmbito municipal & 01 & - & 01 & - & - & 02 \\
\hline \multicolumn{7}{|l|}{ Téenico-assistencial } \\
\hline Ações Educativas & - & - & 01 & - & - & 01 \\
\hline Acolhimento & - & - & 02 & 01 & 01 & 01 \\
\hline Vínculo equipe população / enfoque familiar & 01 & 03 & 05 & 01 & 01 & 07 \\
\hline Integralidade (ações de promoção, prevenção e reabilitação) & 02 & 02 & 05 & - & 01 & 06 \\
\hline Prática clínica & - & - & 01 & - & - & 01 \\
\hline Territorialização & - & 01 & - & - & - & \\
\hline Participação popular & - & 01 & - & - & - & \\
\hline Trabalho em equipe & - & 01 & & - & - & \\
\hline
\end{tabular}

Legenda: * Pequeno porte: <20.000 habitantes; médio > 20.000 e < 100.000 hab. grande porte: acima de 100.000 hab.

tavam relacionados ao PROESF, os quais se constituíram em mais de $50 \%$ dos estudos publicados em 2006 e 2008 (Figura 1).

Quanto ao período em que os dados desses estudos foram coletados, os primeiros ocorreram já em 2000, ou seja, no início da implantação do PSF. 0 período entre 2004 e 2007 foi o que mais teve estudos realizados, ten do como destaque 0 ano de 2005, no qual observou-se maior número de investigações eas quais guardam uma relação com a implementação do PROESF.

No que se refere aos cenários estudados, foram encontrados oito estudos de caso em USF, catorze estudos de caso em um ou dois municípios e dezenove estudos em estratos de três ou mais municípios. Desses últimos, sete foram de senvolvidosa partir do PROESF. Quanto ao porte do município estudado, vinte e três foram em grandes municípios, quatro em municípios de portemédio edois em pequenos municípios. Nos doze restantes, três não especificavam o porte do município e nove tinham, num mesmo estudo, municípios de diferentes portes.
Para obten ção dos dados, 39 estudos utilizaram a entrevista ou grupo focal, sendo $14 \mathrm{com}$ usuários, 27 com profissionais e 20 com gestores. Vale salientar que, do total, 17 utilizaram-se de múltiplos sujeitos, 06 lançaram mão da análise documental e base de dados articulados às entrevistas e 06 usaram a observação como segunda técnica. Em 02 estudos, foi utilizado exclusivamente 0 banco de dados dos Sistemas de Informação.

\section{A mudança do modelo de atenção nos diversos caminhos metodológicos}

Entre os trabalhos analisados, 19 trazem a mudança do modelo como objeto de estudo. Desses, 12 apoiam-seno referencial da Vigilância à Saúde com foco nas necessidades, 05 trabaIham com foco na demanda e 02 não especificam referencial. Dentre os que trabalham com foco na demanda, 02 consideram a mudança do modelo passível deser mensurada quantitativamente pelas atividades dos profissionais, 02 abordam a 
Tabela 3. Distribuição numérica dos artigos sobre a Estratégia Saúde da Família e seu papel na reorientação do modelo de atenção identificados na base de dados Scielo, no período de 2002 e 2010, em categorias que descreveram limites para a mudança do modelo de atenção, segundo as dimensões político-institucional, organizacional e técnico-assistencial, local do estudo e porte do município.

\begin{tabular}{|c|c|c|c|c|c|c|}
\hline \multirow[b]{2}{*}{ Dimensão/categoria } & \multicolumn{3}{|c|}{ Local do estudo } & \multicolumn{3}{|c|}{ Porte do município* } \\
\hline & USF & $\begin{array}{c}1 \text { ou } 2 \\
\text { municípios }\end{array}$ & $\begin{array}{l}3 \text { ou mais } \\
\text { municípios }\end{array}$ & Pequeno & Médio & Grande \\
\hline \multicolumn{7}{|l|}{ Político-institucional } \\
\hline Tipo de Gestão (não participativa) & - & 01 & 06 & 03 & 02 & 06 \\
\hline Política de pessoal & 02 & 03 & 11 & 04 & 05 & 14 \\
\hline Financiamento (insuficiência, dificuldade no fluxo) & - & 02 & 04 & 02 & 04 & 05 \\
\hline Capacidade de governo & 02 & 02 & 08 & 02 & 03 & 10 \\
\hline Capacidade instalada & - & 01 & 03 & 1 & 01 & 04 \\
\hline A ções intersetoriais como política de governo & - & 02 & 06 & 01 & 03 & 06 \\
\hline Projeto de governo (Rotatividade / descontinuidade) & 01 & 04 & 02 & - & - & 07 \\
\hline \multicolumn{7}{|l|}{ Organizacional } \\
\hline Acesso à Atenção Básica ( $n$ o de famílias por equipe) & 01 & 02 & 02 & - & - & 05 \\
\hline Integralidade (acesso aos níveis de atenção) & 01 & 04 & 05 & - & 01 & 08 \\
\hline Articulação entre os níveis do sistema & - & 05 & 04 & - & 02 & 07 \\
\hline Participação popular nas ações locais & 01 & 02 & 05 & 02 & 02 & 06 \\
\hline \multicolumn{7}{|l|}{ Técnico-assistencial } \\
\hline Ações Educativas & 02 & 01 & 02 & 01 & 01 & 05 \\
\hline Acolhimento & 02 & 02 & 03 & 01 & 02 & 03 \\
\hline Vínculo equipe-população / enfoque familiar & 02 & 03 & 04 & 03 & 01 & 06 \\
\hline Integralidade (promoção, prevenção e recuperação) & 04 & - & 10 & 05 & 05 & 14 \\
\hline Prática clínica empobrecida / diversidade das práticas & 01 & 02 & 03 & 02 & 02 & 06 \\
\hline Territorialização & 01 & 01 & 02 & - & - & 03 \\
\hline Estímulo a autonomia e participação popular & 05 & - & 02 & 03 & 01 & 05 \\
\hline Trabalho em equipe & 01 & 04 & 04 & 02 & 03 & 06 \\
\hline
\end{tabular}

Legenda: *Pequeno porte: < 20.000 habitantes; médio $>20.000$ e < 100.000 hab. grande porte: acima de 100.000 hab.

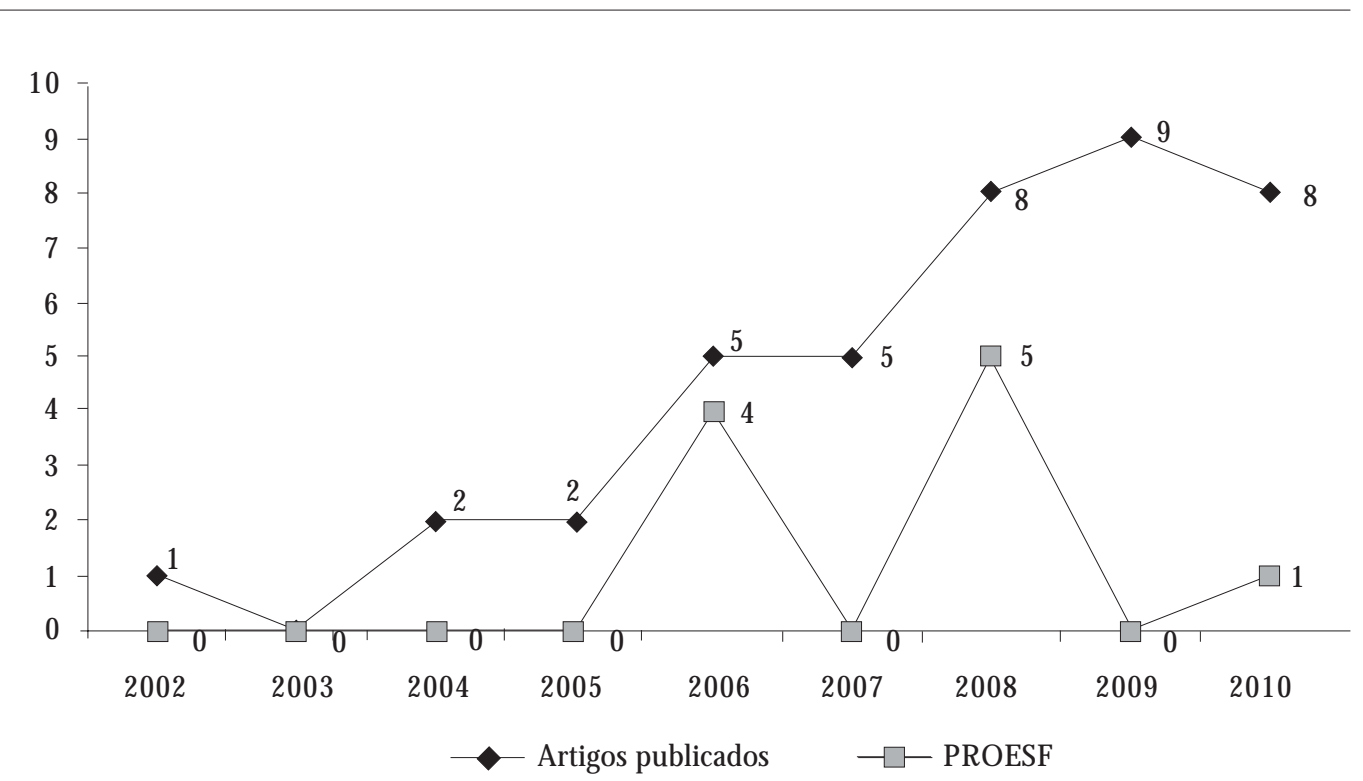

Figura 1. Distribuição dos artigos sobre a Estratégia Saúde da Família e seu papel na reorientação do modelo de atenção, publicados na Base de Dados Scielo entre 2002 e 2010, segundo ano de publicação. 
ESF como modelo de atenção primária e 01 foca 0 acolhimento.

As outras 22 produções analisam componentes do processo de trabalho, sendo que 12 contextualizam o modelo de atenção, estando 07 voltados às necessidades e 05 com foco na demanda. Os demais não fazem referência ao modelo de atenção em seu aporte teórico.

Na grande maioria dos estudos, a concepção da Saúde da Família é apresentada como estraté gia. Somente em um estudo essa concepção não ficou explícita, porém não foi referida também como simples programa. N essa perspectiva, foram encontrados entre os estudos de análise de implementação, 02 em que a implantação do PSF se deu enquanto programa verticalizado. Nos demais, da mesma natureza, foram evidenciados graus de implantação diversificados, mas já na perspectiva de estratégia de mudança do model o.

A categorização dos estudosa partir dos objetos/tipos de estudo está descrita na Tabela 1. Foram predominantes os estudos do grupo 01 que correspondeàs análises deimplantação $(n=18)$ e distribuem-se entre os estudos de avaliação do grau de implantação, estudos de Linhas de Base e outros estudos qualitativos descritivos. Desses, as avaliações do grau de implantação foram mais frequentes, a maioria delas na modalidade de estudos de caso. A penas 02 desses estudos que avaliavam o grau de implantação foram realizados com grandes extratos, os quais utilizaram apenas dados secundários. A maior parte dos artigos que traz resultados dessas avaliações não explicita 0 julgamento de valor do grau de implantação e aqueles que o fazem, mostraram graus de implantação entreincipienteeintermediário.

No grupo 02 , que avalia o processo de trabaIho ou práticas específicas, as práticas mais avaliadas foram aquelas voltadas ao trabalho em equipe ( $n=5$ ). Nesses ainda são evidenciadas fragilidades na inter-relação dos profissionais, principalmente no âmbito do planejamento local e avaliação das ações, ficando focados nas questões burocráticas e operacionais de atendimento à demanda. Nas demais práticas avaliadas, os limites apontados estão relacionados a concepções do modelo biomédico por parte dos sujeitos envolvidos. Os avanços encontrados ainda são no sentido da prática assi stencial, os quais refletem uma melhoria do atendimento individual tendo sido descritas mudanças no acolhimento, vínculo e educação em saúde.
Mudanças nas dimensões

político-institucional, organizacional e técnico-assistencial

As mudanças positivas e os limites para as mudanças têm acontecido nas três dimensões avaliadas, porém nem sempre estão descritas no mesmo estudo. Os estudos em grupos de municípios descrevem um número maior de categorias. (Tabelas 2 e 3 ).

Os estudos em geral apontam um maior número de limites do que de mudanças positivas. Nessas, nenhuma das categorias avaliadas esteve presente em pelo menos $25 \%$ dos estudos, quanto aos limites, os mesmos se aproximam mais desse percentual e em alguns casos até ultrapassam.

As mudanças positivas, quando analisadas por dimensão, apresentam proporções diferentes entre os achados relativos a algumas categorias. As categorias também são descritas em cenários diferentes. A dimensão político-institucional não é avaliada em estudos realizados em USF isolada e predomina nos estudos de grupos de municípios. Entre as categorias se destacam a capacidade instalada, a política de pessoal e o projeto de governo ${ }^{56}$ que priorizam a expansão da ESF. A dimensão organizacional também é pouco frequente nos estudos de caso e as categorias mais descritas são as de acesso à Atenção Básica e a articulação da rede de atenção. Já a dimensão técnico-assistencial encontra-se em maior número entre os estudos de caso de municípios e prevalecem as categorias de integralidade das práticas e vínculo. Tais mudanças, quando relacionadas ao porte dos municípios, estão maisevidenciadasnos municípios degrandeporte (Tabela 2).

Os limites aparecem com suas categorias descritas na maioria dos tipos de cenários, com destaque para aqueles realizados em grandes municípios. A política de pessoal e a capacidade de governo ${ }^{56}$ destacam-sena dimensão político-institucional, a integralidade referente ao acesso aos demais níveis deatenção éevidenciada na dimensão organizacional e a integralidade relativa às práticas e o trabal ho em equipe na dimensão técnico-assistencial (Tabela 3).

Chama atenção a manutenção dos padrões dos achados em todo o período analisado. $\mathrm{Na}$ distribuição temporal das descrições das mudanças e dos limites, deacordo com o ano de estudo, não se verificou uma inversão proporcional. Eles 
aparecem na medida em que os estudos são desenvolvidos, encontrando uma maior frequência de achados no ano de 2005, que foi o ano que mais tiveram pesquisas realizadas.

\section{Discussão}

A ausência de mudanças no modelo de atenção revelada por esse estudo e o grau de implantação daESF ainda incipientea intermediário são reflexo de avanços em alguns aspectos da atenção à saúde, mas que não correspondem ainda à adoção da Saúde da Família como estratégia prioritária e central no nível local, permanecendo a dificuldade em incorporar o caráter substitutivo. Então, mesmo nos locais onde se observa a importância da ESF para garantia do princípio da universalidade, a focalização ainda aparece mascarada, muitas vezes, pelo fato de os extratos de excluídos representarem uma grande proporção populacional ${ }^{57,58}$.

$\mathrm{Na}$ análise da dimensão político-institucional, apontada como explicativa para muitos obstáculos na implementação da ESF ${ }^{23,27}$, observasequea categoria política de pessoal aparecedestacada tanto nas mudanças positivas como nos limites, indicando sua importância nesse processo. Onde ela aparece como mudança, refere-se às estratégias de fixação de profissional ${ }^{28}$, que se constitui em caminho para enfrentar os limites apontados nos outros estudos nos quais a rotatividade de profissionais e as condições de trabaIho precárias apresentaram-se como principais problemas ${ }^{17,18}$.

A formação e a educação permanente se destacam como subcomponentes importantes dessa categoria, onde ainda são presentes os entraves na formação acadêmica que é apontada por alguns estudos como desafio para a ESF ${ }^{18,59}$. Também são referidas as deficiências do aparelho formador dentro do próprio sistema desaúde, principalmenteno nível local, na perspectiva da aprendizagem-trabalho $0^{60}$. A deficiência deprofissionais com perfil para a ESF é descrita pelos estudos, mas em contrapartida é pouco referido o desenvolvimento de ações tanto dos meios instituídos pelas esferas de governo federal eestadual, como outras propostas que viessem a atender às necessidades dos municípios.

Os avanços descritos em relação à capacidade instalada, correspondem ao que os estudos trazem desde 2002 com relação à expansão da saúde da família9,11, até mesmo pelo fato de estar atrelada ao repasse de recursos da atenção básica. En- tretanto, não deixa de aparecer ainda como limites, quando se analisa a ESF, além da concepção de medicina simplificada. Tal situação leva a fazer uma associação com as inconsistências dos projetos de governo, em que a aparente priorização da ESF é confrontada com a descontinuidade da gestão e a rotatividade nos cargos dentro do setor da saúde ${ }^{31,32}$. Somado a isso, a capacidade de governo foi descrita como limites para a ESF ${ }^{33,34}$, pois as mudanças político-institucionais importantes guardaram relações com capacitação da equipe gestora, a boa utilização dos instrumentos de gestão e das informações disponíveis ${ }^{17}$.

A ESF ainda tem refletido o caráter prescritivo de programa ${ }^{61}$, as diferenças nos contextos político, social, econômico e cultural têm resultado muito pouco em estratégia singulares para 0 enfrentamento dos problemas. Q uando analisadas as formas de implementação, estas se caracterizam pelo caráter regional descrito por Viana e Dal $\mathrm{Poz}^{57}$, em que as ações seguem um padrão da esfera estadual, devida à baixa capacidade de formulação local ${ }^{17}$ por parte dos municípios. Exemplo disso é que são pouco descritas políticas intersetoriais desenvolvidas como iniciativas do nível municipal.

0 impacto do caráter ainda incipiente das mudanças políticas leva a relações de trabalho nos moldes antigos. Essas relações reproduzem as antigas práticas na mesma lógica dos modelos hegemônicos, quenão favorecem a mudança nas concepções dos sujeitos, ou seja, a formação que o serviço precisaria proporcionar. Dessa forma, a educação permanente é desenvolvida de maneira dicotômica no que diz respeito à teoria $\mathrm{e}$ prática. A teoria éabordada na lógica dos modelos de atenção alternativos, enquanto na prática os modelos de gestão mantêm a lógica tradicional e os serviços não se constituem espaços de aprendizagem.

Assim, essa fragilidade da dimensão políticoinstitucional reflete diretamente nos limites encontradosna dimensão técnico-assistencial, principalmente nos aspectos relacionados à gestão tradicional que não favorece os espaços democráticos, bem como as políticas sociais. Nos trabalhos analisados, não foram encontrados estudos sobre o desenvolvimento das tecnologias necessárias ao aperfeiçoamento das ações da A tenção Básica, ações intersetoriais e fortalecimento da participação popular.

A gestão tradicional fortalece a cultura da não participação dos profissionais, uma vez que os mesmos não se comportam como usuários do SU Se, nesse tipo degestão, não se colocam como 
sujeitos do processo. Dessa forma, esse comportamento implica na deficiência de práticas nacomunidade que estimulem a participação popular, o que fica evidenciado nos limites apontados em alguns dos estudos ${ }^{15,36,37}$.

No aspecto das políticas intersetoriais, a dimensão político-institucional também implica diretamenteem limites técnico-assistenciais, pois na ausência dessas políticas, a intersetorialidade, queéfundamental para ações de promoção à saúde e para a melhoria da qualidade de vida, tornase uma prática de caráter voluntarioso por parte das equipes ${ }^{58}$ e não conduz aos impactos esperados. Tal circunstância pode levar os profissionais ao descrédito em relação à ESF, sensação de impotência, tendo em vista a incapacidade do setor saúde, isoladamente, enfrentar os determinantes sociais do processo saúde-doença.

A dimensão organizacional foi aqui discutida por ter sido percebida, nesse estudo, como reflexo da dimensão político-institucional e uma via de mão dupla em relação à dimensão técnicoassistencial. A articulação entreosníveis de atenção foi componente importante para aqueles municípios nos quais foram identificadas mudanças nessa dimensão ${ }^{17,19}$. Contudo, para que essa articulação se consolide e a ESF se efetive como porta de entrada para o SUS, é necessário projeto político como projeto de governo, capacidade instalada e conhecimento técnico por parte dos gestores.

Da mesma forma, a melhoria descrita no acesso à Atenção Básica, muitas vezes não vem acompanhada da melhoria no acesso aos serviços especializados ${ }^{31}$, fato quepode estar relacionado aos limites de financiamento ou à fragilidade no projeto de governo; também sofre as consequências do uso inadequado dos níveis de maior complexidade devido às práticas clínicas empobrecidase excessivamente dependentes de exames complementares ${ }^{32}$ e à deficiência de ações que integram promoção, prevenção e recuperação ${ }^{19,36}$.

Quanto aos espaços de controle social, pode ser feita análise bem semel hante. 0 tipo de gestão contribui para uma maior ou menor participação da sociedade no processo decisório. Por outro lado, o conhecimento precisa ser produzido nos espaços da comunidade, pois, se esta não conhece o sistema, ela não terá condições de contribuir para sua consolidação. Logo, é contraditória uma proposta que tem a participação popular como um de seus eixos estruturantes permanecer com práticas educativas nos moldes da pedagogia tradicional e quenão tenha a temática sobre a própria estratégia abordada nas ações educativas, como foi demonstrado por alguns autores ${ }^{36}$.

Diante disso, a integralidadetalvez seja o princípio do SUS mais comprometido nessas fragilidades e o que diretamente necessita de mudanças nas três dimensões para que seja efetivada. Afinal, para garantia desse princípio fazem-se necessárias uma clínica abrangente e uma integração de práticas individuais e coletivas no âmbito da promoção, prevenção e recuperação, que são aspectos técnico-assistenciais. N ecessitaigualmentederesolutividade com garantia deacesso e articulação dos níveis de atenção no âmbito organizacional. Para tanto, essa capacidade instalada da rede, bem como as ações intersetoriais requerem ações no âmbito da gestão nas três esferas de governo, permeadas pela participação popular62.

Um aspecto importante a ser destacado no estudo é que a proporção nas categorias descritas não é, necessariamente, a frequência em que os eventos acontecem. Ela repercute os objetos eleitos para estudo, principalmente aqueles em que estudavam práticas isoladas. 0 mesmo se estende para os cenários nos quais essas categorias foram evidenciadas. Embora o fato de os limites aparecerem em proporções maiores e melhor distribuídos nos diferentes locais de estudos, refletiriam possivelmenteuma maior prevalência dos limites em relação às mudanças positivas no contexto geral.

Outro aspecto relevante foi o baixo grau de conhecimento produzido referente a algumas categorias. Muitas delas só apareceram em estudos de grandes extratos, assim, tem-se a vantagem da possibilidade de generalizações. Contudo, perde-se no aprofundamento que contribui para identificar as possi bilidades de enfrentamento. Tem-se, por exemplo, a dimensão políticoinstitucional pouco explorada em estudos decaso singulares. Por outro lado, as estratégias de territorialização, o trabalho em equipe e a participação popular foram pouco descritos nos estudos com cenários mais abrangentes.

M as o maior limite verificado neste estudo, diz respeito à consistência do referencial de modelo de atenção identificado em muitos trabaIhos, que dificulta a categorização entre abordagem por demanda ou necessidade. Pelo menos nos artigos, muitos não foram explícitos, podendo têlo feito nos trabalhos na íntegra. Além disso, mesmo que al guns deles tivessem fundamentado em seu referencial o foco nas necessidades, ao apresentar seus resultados, estes estavam voltados para a demanda. 
M esmo com os limites apontados, verificouse também evidências de aspectos que se mostraram potencializadores de mudanças como a melhoria da capacidadedegestão, política de pessoal, as políticas intersetoriais e a articulação entre os níveis de atenção apontados em al guns estudos. São retratados igualmente aspectos que ainda desafiam a implementação da estratégia como a integralidade de práticas; trabalho em equipe; territorialização; educação em saúde; vínculo no sentido de responsabilização; participação popular e acesso aos serviços de média e alta complexidade.

\section{Conclusão}

A implementação da ESF no Brasil tem desencadeado mudanças no processo de trabalho em saúde, todavia tem selimitado ao âmbito da atenção básica na maioria das realidades estudadas. Os model os hegemônicos continuam exercendo grande influência nas práticas da ESF e principalmente na organização da rede de atenção. Logo, a saúde da família tem sido estratégia de expansão de cobertura, universalização, muitas vezes de forma focalizada e têm sido ainda limitadas as experiências substitutivas.

Em virtude das grandes diferenças sociopolíticas e sua relação com o grau de organização dos serviços e as necessidades em saúde, percebe-se uma carência de estudos que reflitam a re alidade em municípios de pequeno e médio porte, principalmente estudos de grandes extratos. Astrês dimensões aqui analisadas ainda são pouco exploradas de forma conjugada nos estudos avaliativos, tendo sido revelada apenas nos Estudos de Linha de Base do PROESF.

Observaram-se avanços na capacidade instalada, projetos de governo que priorizam a Saúde da Família, melhoria do acesso à atenção básica, estabelecimento de vínculo entre equipe e população e incremento de ações preventivas. A pesar disso, limites importantes se apresentam para que a implementação da ESF venha cumprir seu papel reorientador da mudança do modelo deatenção. Destaca-se aqui a política de pessoal, a capacidade de governo, políticas intersetoriais no nível local, integralidade da atenção e das práticas, territorialização e participação popular. Foi evidente quetanto as mudanças verificadas como os limites se distribuíram nas três dimensões analisadas.
Este estudo se limitou à análise das publicações nacionais em uma única base de dados 0 que compromete sua capacidade de generalização, ainda que as evidências dos trabalhos primários tenham sido descritas e analisadas. Suge rem-se avaliações da ESF que permitam identificar as diferenças regionais para orientar 0 enfrentamento de problemas condizentes com cada realidade. Entretanto, uma grande diversidade metodológica dos estudos e as diferentes concepções quanto ao modelo de atenção impossibilitam maiores generalizações dos resultados encontrados.

Portanto, faz-se necessário também avançar em pesquisas avaliativas que visem diminuir as diferenças metodológicas, mas sem perder de vista a possibilidade de severificar as singularidades.

\section{Colaboradores}

LA Silva coletou os dados, analisou e escreveu o manuscrito. CA Casotti contribuiu com a discussão, redação e revisão final do artigo. SCL Chaves contribuiu com a discussão, redação e revisão final do artigo.

\section{Agradecimentos}

Os agradecimentos vão para os colegas da disciplina Oficina de artigos para a Área de Política, Planejamento e Gestão do Instituto de Saúde Coletiva da U FBA que contribuíram na concepção inicial deste trabalho. De maneira especial, agradecemos ainda à professora Ligia M aria Vieira da Silva que, na condição de docente dessa disciplina, deu preciosas e sábias contribuições. Por fim, agradecemos também à professora Vera Pacheco pela revisão ortográfica. 


\section{Referências}

1. Teixeira CF. Saúde da Família, Promoção e Vigilância: construindo a integralidade da atenção à saúde no SUS. In: Teixeira CF, Solla JP, organizadores. M odelo de Atenção à Saúde: promoção, vigilância e saúde da família. Salvador: Edufba; 2006.

2. Giovanella $L, M$ endonça $M H M$. Atenção Primária à Saúde. In: Giovanella L, Escorel S, Lobato LVC, Noronha JC, Carvalho AI, organizadores. Políticas e Sistema de Saúde no Brasil. Rio de Janeiro: Editora Fiocruz; 2008.

3. Teixeira CF. A Mudança do Modelo de Atenção à Saúde no SUS: desatando nós, criando laços. In: Teixeira CF, Solla JP, organizadores. M odelo de Atenção à Saúde: promoção, vigilância e saúde da família. Salvador: Edufba; 2006.

4. Paim JS. Modelos de Atenção à Saúde no Brasil. In: Giovanella L, Escorel S, Lobato LVC, Noronha JC, Carvalho Al, organizadores. Políticas e Sistema de Saúde no Brasil. Rio de Janeiro: Editora Fiocruz; 2008.

5. Brasil. Ministério da Saúde (MS). Secretaria de Assistência à Saúde. Coordenação de Saúde da Comunidade (MS). Saúde da Família: uma estratégia para a reorientação do modelo assistencial. Brasília: M S; 1997.

6. Brasil. M inistério da Saúde (MS). Secretaria de Atenção à Saúde. Departamento de Atenção Básica. Política Nacional de Atenção Básica. 4a Edição. Braślia: M S; 2007. (Série Pactos pela Saúde 2006; v. 4)

7. Ronzani TM, Stralen CJ. Dificuldades de Implantação do Programa de Saúde da Família como Estratégia de Reforma do Sistema de Saúde Brasileiro. Revista APS 2003; 6(2):99-107.

8. Sousa M F, Hamann EM. Programa Saúde da Família no Brasil: uma agenda incompleta? Cien Saude Colet 2009; 14(Supl.1):1325 -1335.

9. Pereira RAG. Programa Saúde da Família: determinantes e efeitos de sua implantação em municípios brasileiros [tese]. Salvador (BA): Instituto de Saúde Coletiva, Universidade Federal da Bahia; 2006.

10. Brasil. M inistério da Saúde (MS). Secretaria de Atenção à Saúde. Departamento de Atenção Básica. Saúde da família no Brasil: uma análise de indicadores selecionados: 1998-2004. Brasília: M S; 2006.

11. Brasil. M inistério da Saúde (MS). Secretaria de Atenção à Saúde. Departamento de Atenção Básica. Avaliação normativa do Programa Saúde da Família no Brasil: monitoramento da implantação e funcionamento das equipes de saúde da família: 2001-2002. Brasília: M S; 2004.

12. Hartz ZM, Contadriopoulos AP. Do Quê ao Pra Quê da M eta-Avaliação em Saúde. In: Hartz ZM. M eta-avaliação da Atenção Básica à Saúde - teoria e prática. Rio de Janeiro: Editora Fiocruz; 2008.

13. Almeida PF, Giovanella L. Avaliação em Atenção Básica à saúde no Brasil: mapeamento e análise das pesquisas realizadas e/ou financiadas pelo $\mathrm{M}$ inistério da Saúde entre os anos de 2000 e 2006. Cad Saude Publica 2008; 24(8):1727-1742.

14. Lentsck MH, Kluthcovsky ACGC, Kluthcovsky FAK. Avaliação do Programa Saúde da Família: uma revisão. Cien Saude Colet 2010; 15(Supl.3):3455-3466.

15. Lacerda WA, Santiago IM FL. A participação popular na gestão local do Programa Saúde da Família em Campina Grande, Paraíba. Rev Katálysis 2007; 10(2):197-205.
16. Vanderlei MIG, Almeida M CP. A concepção e prática dos gestores e gerentes da estratégia de saúde da família. Cien Saude Colet 2007; 12(2):443-453.

17. Rocha PM, Uchoa AC, Rocha NSPD, Souza ECF, Rocha ML, Pinheiro TXA. Avaliação do Programa Saúde da Família em municípios do N ordeste brasileiro: velhos e novos desafios. Cad Saude Publica 2008; 24(Supl.1):69-78.

18. Camargo Júnior KR, Campos EAS, Bustamante-Teixeira MT, Mascarenhas MTM, M auad NM, Franco TB, Ribeiro LC, Alves M JM. Avaliação da atenção básica pela ótica político-institucional e da organização da atenção com ênfase na integralidade. Cad Saude Publica 2008; 24(Supl.1):58-68.

19. Escorel S, Giovanella L, M endonça MHM, Senna M CM . O Programa de Saúde da Família e a construção de um novo modelo para a atenção básica no Brasil. Rev Panam Salud Publica 2007; 21(2):164-176.

20. Souza MF, Merchán-Hamann E. Saúde da Família no Brasil: estratégia de superação da desigualdade na saúde? Physis Rev Saúde Coletiva 2009; 19(3):711-729.

21. Silva NC, Garnelo L, Giovanella L. Extensão de Cobertura ou Reorganização da Atenção Básica? A trajetória do Programa de Saúde da Família de M anaus-AM. Saude Soc 2010; 19(3):592-504.

22. Stralen CJ, Belisário SA, Stralen TBS, Lima AMD, Massote AW, Oliveira CL. Percepção dos usuários e profissionais de saúde sobre atenção básica: comparação entre unidades com e sem saúde da família na Região Centro-O este do Brasil. Cad Saude Publica 2008; 24(Supl.1):148-158.

23. Viana AL, Rocha JSY, Elias PE, I báñez $N$, N ovaes MHD. Modelos de atenção básica nos grandes municípios paulistas: efetividade, eficácia, sustentabilidade e governabilidade. Cien Saude Colet 2006; 11(3):577-606

24. Fracolli LA, Zoboli ELCP. Descrição e análise do acolhimento: uma contribuição para o programa de saúde da família. Rev Esc Enferm USP 2004; 38(2):143-151.

25. Schimith MD, Lima MADS. Acolhimento e vínculo em uma equipe do Programa Saúde da Família. Cad Saude Publica 2004; 20(6):1487-1494.

26. Silva IZQJ, Trad LAB. 0 trabalho em equipe no PSF: investigando a articulação técnica e a interação entre os profissionais. Interface Comun Saúde Educ 2005; 9(16):25-38.

27. M edina M G, H artz ZM. The role of the Family $\mathrm{H}$ ealth Program in the organization of primary care in municipal health systems. Cad Saude Publica 2009; 25(5):1153-1167.

28. M elo EM, Paiva L, Álvares J, Flecha ALD. A organização da Atenção Básica em municípios integrantes do Projeto de Expansão e Consolidação do Saúde da Família em M ato Grosso, Brasil. Cad Saude Publica 2008; 24(Supl.1):29-41.

29. Copque HLF, Trad LAB. Família: a experiência de implantação em dois M unicípios da Bahia. Epidemiol. Serv. Saude 2005; 14(4):223-233.

30. Giacomozzi CM, Lacerda MR. A prática da assistência domiciliar dos profissionais da Estratégia de Saúde da Família. Texto Contexto Enferm 2006; 15(4):645-653. 
31. Kantorski LP, Jardim VM R, Coimbra VCC, Oliveira M M, Heck RM. A integralidade da atenção à saúde na perspectiva da gestão no município. Texto Contexto Enferm 2006; 15(3):434-441.

32. Shimizul HE, Rosales C. As práticas desenvolvidas no Programa Saúde da Família contribuem para transformar o modelo de atenção à saúde? Rev Bras Enferm 2009; 62(3):424-429.

33. Facchini LA, Piccini RX, Tomasi E, Thumé E, Silveira DS, Siqueira FV, Rdrigues MA. Desempenho do PSF no Sul e no Nordeste do Brasil: avaliação institucional e epidemiológica da Atenção Básica à Saúde. Cien Saude Coletiva 2006; 11(3):669-681.

34. Veras CLS, Vianna RPT. Desempenho de municípios paraibanos segundo avaliação de características da organização da atenção básica - 2005. Epidemiol Serv Saude 2009; 18(2):133-140.

35. Bousquat A, Cohn A, Elias PE. Implantação do Programa Saúde da Família e exclusão sócio-espacial no M unicípio de São Paulo, Brasil. Cad. Saude Publica 2006; 22(9):1935-1943.

36. Besen CB, Souza N eto M. A Estratégia Saúde da Família como objeto de educação em saúde. Saude soc 2007; 16(1):57-68.

37. Campos L, Wendhausen A. Participação em saúde: concepções e práticas de trabalhadores de uma equipe da estratégia de saúde da família. Texto Contexto Enferm 2007; 16(2):271-279.

38. Canesqui AM, Spinelli MAS. A implementação do Programa Saúde da Família em municípios do Estado de M ato Grosso, Brasil. Cad Saude Publica 2008; 24(4):862-870.

39. Souza ECF, Vilar RLA, Rocha NSPD, Uchoa ACU, Rocha PM. Acesso e acolhimento na atenção básica: uma análise da percepção dos usuários e profissionais de saúde. Cad Saude Publica 2008; 24 (Supl. 1):100110.

40. Henrique F, Calvo M CM . Avaliação do Programa Saúde da Família nos municípios do Estado de Santa Catarina, Brasil. Cad. Saude Publica 2008; 24(4):809-819.

41. Souza M F. O Programa Saúde da Família no Brasil: análise do acesso à atenção básica. Rev Bras Enferm 2008; 61(2):153-158.

42. Conill EM. Políticas de atenção primária e reformas sanitárias: discutindo a avaliação a partir da análise do Programa Saúde da Família em Florianópolis, Santa Catarina, Brasil, 1994-2000 Cad Saude Publica 2002; 18(Supl):191-202.

43. Pavoni DS, Medeiros CRG. Processos de trabalho na Equipe Estratégia de Saúde da Família. Rev Bras Enferm 2009; 62(2):265-271.

44. Giovanella L, M endonça M H M, Almeida PF, Escorel $S$, Senna MCM, Fausto MCR, Delgado M M , Andrade CLT, Cunha MS, Martins MIC, Teixeira CP. Saúde da família: limites e possibilidades para uma abordagem integral de atenção primária à saúde no Brasil. Cien Saude Colet 2009; 14(3):783-794.

45. Kantorski LP, Jardim VMR, Pereira DB, Coimbra VCC, Oliveira M M. A integralidade no cotidiano de trabalho na Estratégia Saúde da Família. Rev Gaúcha Enferm 2009; 30(4):594-601.

46. Trad LAB, Esperidião MA. Gestão participativa $e$ corresponsabilidade em saúde: limites e possibilidades no âmbito da Estratégia de Saúde da Família. Interface Comun Saude Educ 2009; 13(Supl.1):557-570.
47. Junges JR, Selli L, Soares NA, Fernandes RBP, Schre ck M. Processos de trabalho no Programa Saúde da Família: atravessamentos e transversalidades. Rev Esc Enferm USP 2009; 43(4):937-944.

48. Silva JM, Caldeira AP. M odelo assistencial e indicadores de qualidade da assistência: percepção dos profissionais da atenção primária à saúde. Cad Saude Publica 2010; 26(6):1187-1193.

49. Serra CG, Rodrigues PHA. Avaliação da referência e contrarreferência no Programa Saúde da Família na Região M etropolitana do Rio de Janeiro (RJ, Brasil). Cien Saude Colet 2010; 15(Supl. 3):3579-3586.

50. Azevedo ALM, Costa AM. A estreita porta de entrada do Sistema Ú nico de Saúde (SUS): uma avaliação do acesso na Estratégia de Saúde da Família. Interface Comun Saúde Educ 2010; 14(35):797-810.

51. Marqui, ABT, Jahn AC, Resta DG, Colomé ICS, Rosa N, Zanon T. Caracterização das equipes da Saúde da Família e de seu processo de trabalho. Rev Esc Enferm USP 2010; 44(4):956-961.

52. Albuquerque FJB, M elo CF. Avaliação dos Serviços Públicos de Saúde em duas capitais nordestinas do Brasil. Psic.: Teor. e Pesq. 2010; 26(2):323-330.

53. Figueiredo PP, Cezar-Vaz MR, Soares JFS, Sena J, Cardoso LS. Processo de trabalho da Estratégia Saúde da Família: a concepção de gestão que permeia o agir em saúde. Physis 2010; 20(1):235-259.

54. Kell MCG, Shimizu HE. Existe trabalho em equipe no Programa Saúde da Família? Cien Saude Colet 2010; 15(Supl.1):1533-1541.

55. Oliveira HM, Gonçalves MJF, Pires ROM. Caracterização da Estratégia Saúde da Família no Estado do Amazonas, Brasil: análise da implantação e impacto. Cad Saude Publica 2011; 27(1):35-45.

56. Matus C. Política, Planificação e Governo. 2a Edição. Brasília: IPEA; 1993.

57. Viana AL, Dal Poz MR. A Reforma do Sistema de Saúde no Brasil e o Programa de Saúde da Família. Physis Rev Saude Coletiva 2005; 15(Supl):225-264.

58. Orazem CA, Camargo Júnior KR. Alguns desafios conceituais e técnico-operacionais para o desenvolvimento do Programa de Saúde da Família como uma proposta transformadora do modelo assistencial. Physis Rev Saude Coletiva 2002; 12(1):59-75.

59. M endonça CS. Saúde da Família, agora mais do que nunca! Cien Saude Colet 2009; 14(Supl.1):1493-1497.

60. Pessanha RV, Cunha FTS. A aprendizagem-trabaIho e as tecnologias de saúde na estratégia saúde da família. Texto Contexto Enferm 2009; 18(2):233-240.

61. Ribeiro EM, Pires D, Blank VLG. A teorização sobre processo de trabalho em saúde como instrumental para análise do trabalho no Programa Saúde da Família. Cad Saude Publica 2004; 20(2):438-446.

62. Paim JS. Atenção à saúde no Brasil. In: Brasil. M inistério da Saúde (MS). Saúde no Brasil: contribuições para a Agenda de Prioridades de Pesquisa. $2^{\underline{a}}$ Edição. Brasília: MS; 2006.

Artigo apresentado em 03/06/2011

A provado em 20/05/2011

Versão final apresentada em 25/05/2011 